Document downloaded from:

http://hdl.handle.net/10251/58588

This paper must be cited as:

Salazar Afanador, A.; Safont Armero, G.; Vergara Domínguez, L. (2011). Application of independent component analysis for evaluation of ashlar masonry walls. Lecture Notes in Computer Science. 6691(1):469-476. doi:10.1007/978-3-642-21498-1_59.

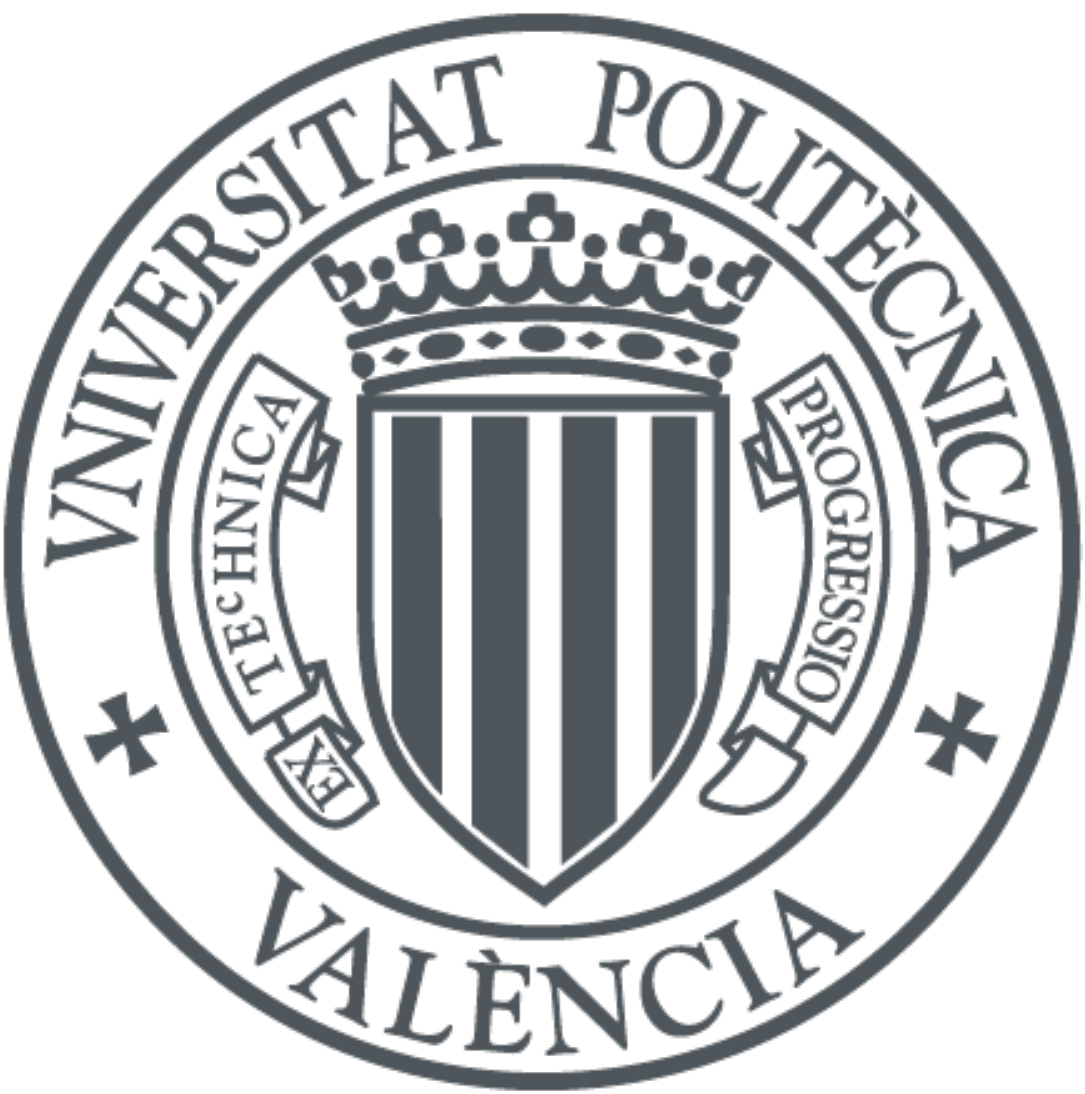

The final publication is available at

http://dx.doi.org/10.1007/978-3-642-21498-1_59

Copyright Springer Verlag (Germany)

Additional Information 


\title{
Application of Independent Component Analysis for Evaluation of Ashlar Masonry Walls
}

\author{
Addisson Salazar $^{1}$, Gonzalo Safont ${ }^{1}$, Luis Vergara ${ }^{1}$ \\ ${ }^{1}$ Instituto de Telecomunicaciones y Aplicaciones Multimedia, Universidad Politécnica de Valencia, Camino de Vera \\ $\mathrm{s} / \mathrm{n}, 46022$, Valencia, Spain \\ asalazar@dcom.upv.es, gonsaar@upvnet.upv.es, Ivergara@dcom.upv.es
}

\begin{abstract}
This paper presents a novel application of Independent Component Analysis (ICA) to the evaluation of ashlar masonry walls inspected with Ground Penetrating Radar (GPR). ICA is used as preprocessor to eliminate the background from the backscattered signals and thus signal-to-noise ratio of the GPR signals is enhanced. Several experiments were made on scale models of historic ashlar masonry walls. These models were loaded with different weights, and the corresponding BScans were obtained. ICA shows the best performance to enhance the quality of the B-Scans compared with classical methods used in GPR signal processing.
\end{abstract}

Keywords: ICA, GPR, Clutter, NDT

\section{Introduction}

Non-destructive testing (NDT) has been supported by computational intelligent methods such as neural networks [1] and independent component analysis (ICA) [2]. The use of ICA for Ground Penetrating Radar (GPR) signal processing has been recently studied in [3], [4], [5], [6]. Most of these works deal with the use of stepped-frequency GPR for the detection of nonmetallic land mines. In this paper, a novel application of ICA to Non-Destructive Testing (NDT) of historical walls using GPR is presented. The overall objective is to reduce the clutter in the captured GPR signal in order to enhance the radargrams of the wall internal structure. A radargram is an image that represents values of the measured signals at different depths of the material through the points of a trajectory used to examine the material. Each of the signals collected is called an "A-Scan" and the map formed by the collection of all the A-Scans in a trajectory is called a "B-Scan" (i.e., the radargram); see examples of B-Scan in Fig. 4.

Two scale models of historical ashlar masonry walls were analyzed: the first one was homogeneous and the second one was previously mechanized to create imperfections in specific locations. We will show the detection of these inhomogeneities inside the wall and the characterization of the propagation of electromagnetic waves inside the masonry under different loads. Fig. 1 shows an outline of the walls that depicts the following: ashlars, mortar interfaces between ashlars, imperfections, and trajectories of the GPR data acquisition (7 columns and 4 rows). The walls were $2.87 \mathrm{~m} . \times 2.2 \mathrm{~m} . \times 0.204 \mathrm{~m}$. (width, height and length). 


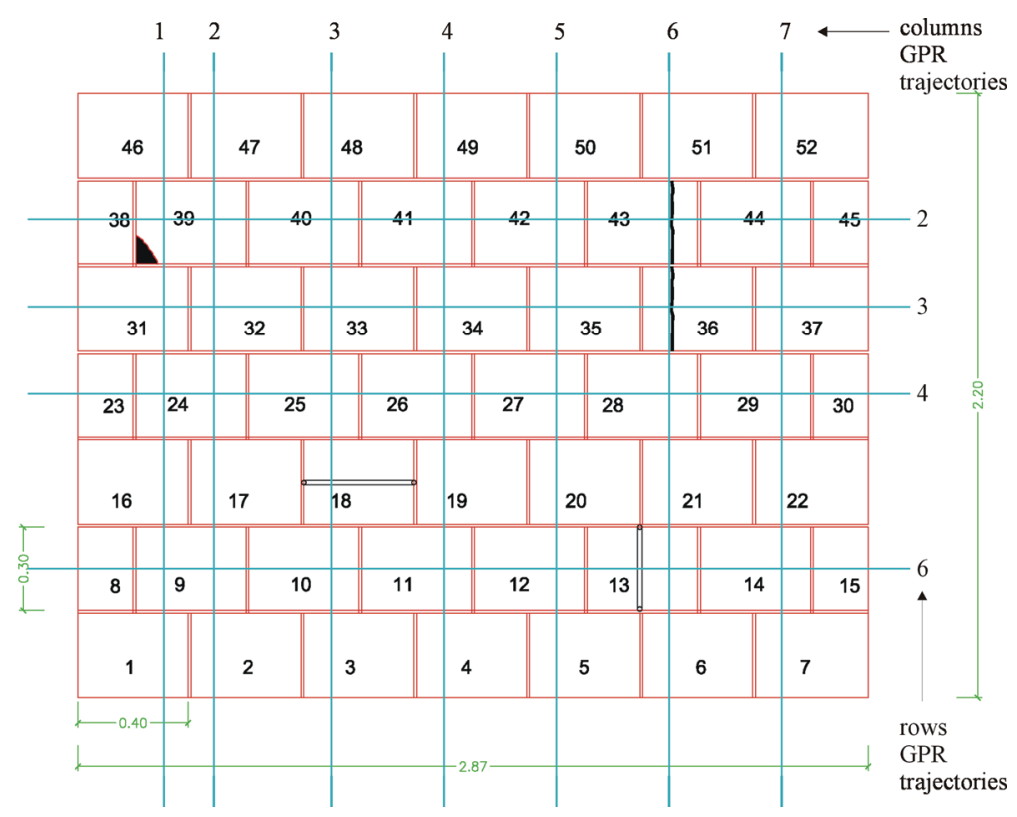

Fig. 1. Geometry of the wall under test. Ashlars, mortar interfaces and imperfections are depicted.

An ICA model for the received GPR signals that allows the background component from the backscattered components to be separated is proposed. The background component was used to assess the deformation of the wall under a load applied at the centre of the top of the wall using hydraulic pressure. The backscattered components were analyzed to detect the imperfections inside the wall. The algorithms employed for background estimation at the first step of the GPR signal processing were the following: (i) ICA algorithms - Mixca [7], JADE [8], TDSEP [9]; (ii) polynomial estimation; and (iii) spatial mean [10].

\section{Statement of the ICA Problem}

The final objective of this application is to obtain clear maps (B-Scans) of a wall inspected by GPR that allow the imperfections inside the wall to be detected. Thus, we propose ICA for a preprocessing step to separate the so-called background (reflections from the air-wall interfaces) from the rest of the backscattered measured signal. The background is a kind of interference that has to be removed in order to enhance the signal-to-noise ratio (SNR) of the GPR signals [11]. Thus, we considered the measured signal as a mixture obtained from the backscattering of the material microstructure plus the background.

The signal of backscattering measured by GPR, under assumptions on the wavelength and the scattering size, can be modelled as a stochastic process. This model is composed of a homogeneous non-dispersive media, and randomly distributed punctual scatters depicting the composite nature of the received grain noise signal instead of a rigorous description of the material microstructure. Thus, the backscattering model can be written as [12],

$$
\{\tilde{Z}(p, t)\}=\sum_{n=1}^{N(p)} \tilde{A}_{n, p} f\left(t-\tilde{\tau}_{n}(p)\right)
$$


where $p$ is the location of the GPR antenna in the B-Scan. The random variable (r.v.) $\tilde{A}_{n, p}$ is the scattering cross-section of the $n$th scatter in the location $p$. The r.v. $\tilde{\tau}_{n}$ is the delay of the signal backscattered by the $n$th scatter and $N(p)$ is the number of scatters contributing from this position. The function $f(t)$ is the pulse emitted by the GPR, which is defined as

$$
f(t)=b \cdot\left(1-\left(\omega_{0} t\right)^{2}\right) e^{\frac{-\left(\omega_{0} t\right)^{2}}{2}}
$$

where $\omega_{0}$ is the central frequency of the GPR antenna (in this application it was $1.6 \mathrm{GHz}$ ) and $b$ is a normalization factor.

The recorded signals can be modelled as the superposition of the backscattered signal plus sinusoidal phenomena representing the background. The extraction of the background was made using a sliding window of size 100 A-Scans with an overlap of 99 A-Scans. For each window, a background was estimated using ICA. This can be written as,

$$
x_{k, l}(t)=s_{k, l}(t)+\sum_{i=1}^{N-1} \alpha_{i k l} e^{j\left(\omega_{i} t+\theta_{i k}\right)} \quad k=1 \ldots M \quad l=1 \ldots L
$$

where $M$ is the number of A-Scans, $L$ is the number of windows, $x_{k, l}(t)$ is the received signal from the material at position $k$ of the B-Scan estimated in the window $l ; s_{k, l}(t)$ is the backscattering signal that depends on the material microstructure; and $\alpha_{i k l} e^{j\left(\omega_{i}+\theta_{i k}\right)} i=1 \ldots N-1, k=1 \ldots M, l=1 \ldots L$ are the sinusoidal sources to be analyzed with $\alpha$ amplitude, $\omega$ angular frequency, and $\theta$ initial phase.

In the proposed application, the wall was scanned for 7 columns (vertical scan) and 4 rows (horizontal scan), see Fig. 1. Due to constraints for the movement of the antenna survey wheel, actual length of the acquisition trajectories was $1.7 \mathrm{~m}$. and $2.1 \mathrm{~m}$. for columns and rows respectively. The scan density was 200 A-Scans per metre and 1024 samples were acquired for each A-Scan. Thus, $M=340$ for vertical scanning and $M=420$ for horizontal scanning. This number of scanning was adequate to include the anomalies of the material and background. Background estimation is performed for each window and thus its performance is not affected by the sampling configuration. On the contrary, B-Scan resolution depends on the number of data available to build the wall image, even though this resolution can be enhanced by using interpolated data. The suitability of this sampling configuration for the present application is demonstrated in Section 4 (Results and Discussion).

Obviously, the estimated sinusoidal components have the same frequencies along the B-Scan, with possibly changing amplitude and phase. From a statistical point of view, considering the background as a sinusoid with deterministic but unknown amplitude and uniform random phase, it is clearly guaranteed that the backscattering signal and the background are statistically independent. Therefore, ICA algorithms can separate the background (characterized for one sinusoidal source estimated by ICA) from the backscattering (the rest of sources estimated by ICA) contribution. 


\section{Performance Analysis of Background Estimation Methods}

Historic walls suffer degradation of its physical properties with the pass of time. This can generate a strong clutter in the measured signals using GPR. For this work, two walls were only available with particular SNR. Thus, we generated more cases of historic walls in different conditions by adding K-distributed noise to the measured signals. K-distribution has been demonstrated to be a good model for radar clutter [13]. The SNRs were 0, 2, 4, 6, 8, 10, 15, 20, $25,30,40,50,60 \mathrm{~dB}$. For each different SNR, the background was estimated using the following methods: ICA algorithms (JADE, Mixca, and TDSEP), polynomials, and spatial mean, see Fig. 2.

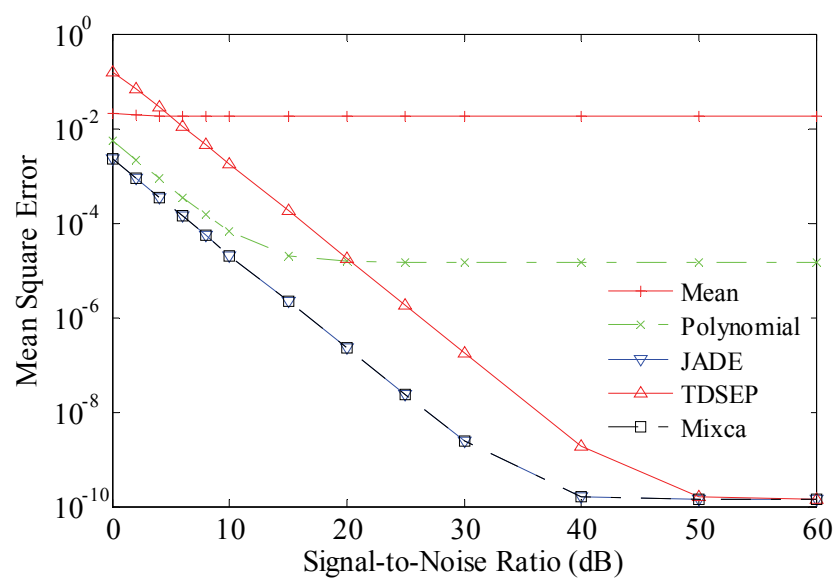

Fig. 2. Performance analysis of different methods for background estimation.

Fig. 2 shows the Mean Square Error (MSE) between the background of one of the original Bscans and the background estimated with added K-distributed noise. JADE and Mixca achieve the best results, i.e. the background is well-estimated even for low SNRs. ICA methods show similar behaviours, improving the estimation with higher SNR, while classical methods (polynomial, mean) do not improve significantly. The separation of the source corresponding to the background with any small energy level is possible using ICA since it is based on statistical independence of the components and not on the energy associated to each frequency component. We selected the Mixca algorithm for the preprocessing step. This algorithm implements non-parametric source density estimation, which has allowed its application to different applications such as NDT [2][14] and biosignal processing [15]. Fig. 3 shows one of the sources (ICA component) obtained for the background. 


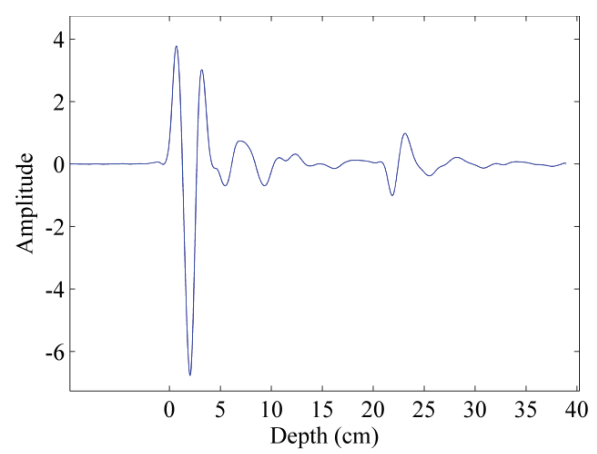

Fig. 3. Source signal corresponding to the background estimated in a single window.
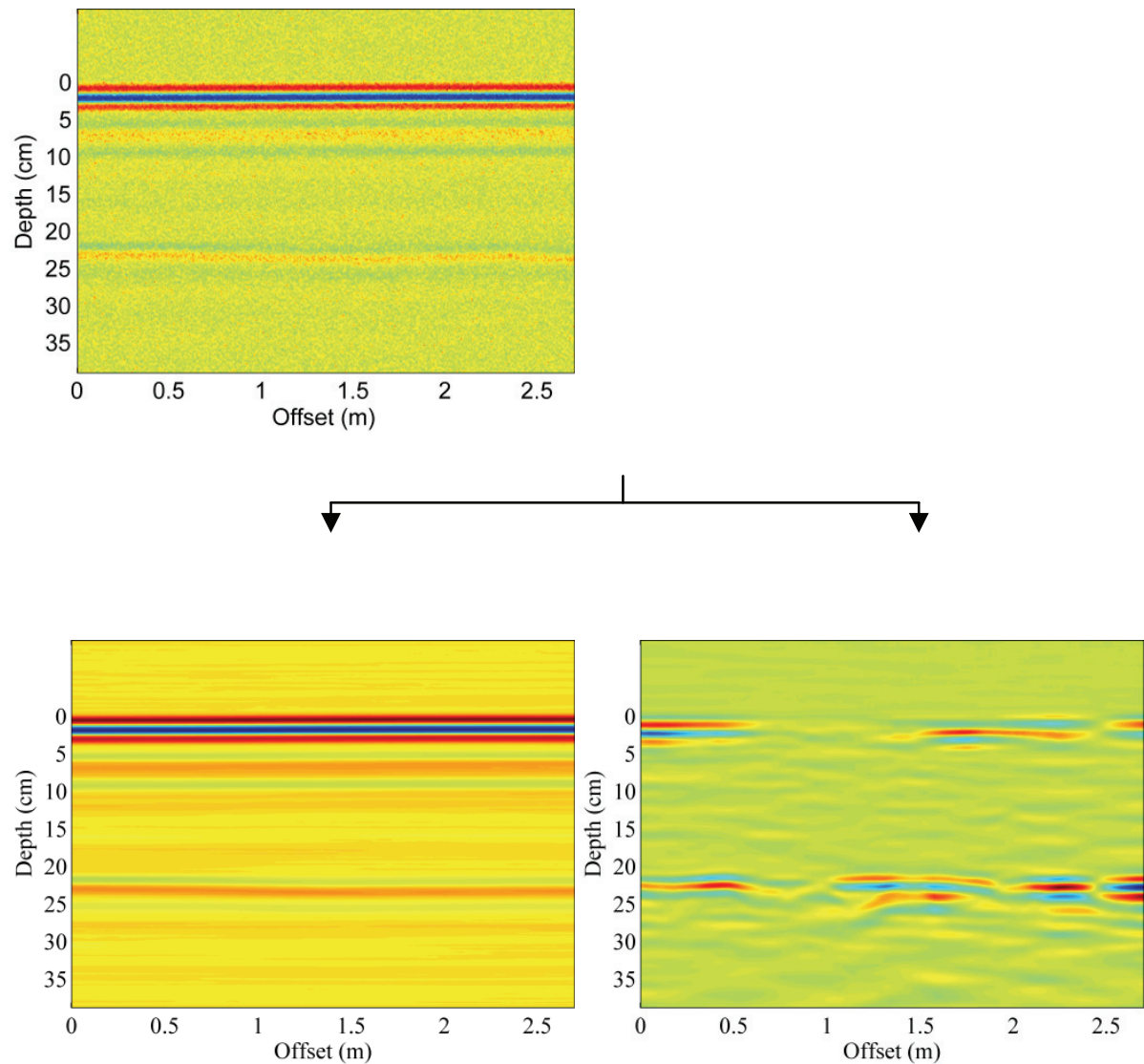

Fig. 4. B-Scan from GPR signals added with K-distributed noise for $0 \mathrm{~dB}$ SNR (upper graph); estimated background component (lower left graph); backscattered components (lower right graph).

Fig. 4 shows the separation of the background component and the backscattered components from the GPR measured signals with $0 \mathrm{~dB}$ SNR. The pulse generated by the GPR equipment propagates from the antenna to the wall through an air interface. After this, the pulse travels through the wall until the opposite side of the wall. Finally, it keeps propagating through free air. The signal reflected from these two interfaces forms the background component. Grain noise and imperfections in the wall's structure form the backscattered components. Note that it is very difficult to obtain any information about the wall's condition from the graph in the upper part of Fig. 4. However, some locations of interest are clearly depicted in the lower right graph of Fig. 4. In the next section, we will show that there were imperfections in the wall located in these locations. 


\section{$4 \quad$ Results and Discussion}

The equipment employed consisted of a GPR system, SIR 3000 from Geophysical Survey Systems Inc. We used a $1.6 \mathrm{GHz}$ mounted on an encoder. The receiving antenna had a size of $3.8 \mathrm{~cm} . \times 10 \mathrm{~cm} . \times 16.5 \mathrm{~cm}$ that was adequate for both vertical and horizontal measures. The configuration parameters used for data acquisition were: distance mode (200 scans per meter), range of $10 \mathrm{~ns}$ and 1024 samples per scan. In order to obtain enhanced B-Scans of the wall.

\subsection{Analysis of the Homogeneous Wall (Background Component)}

Fig. 5 shows the background signals of captured radargrams for the homogeneous ashlar masonry wall, for two different loads. A variation of the propagation conditions of the wall is shown. Note the way the opposite side of the wall seems to move away (see Fig. 5.b) that indicates a loss in velocity of propagation inside the material. Thus, worsening of transmission properties in the wall was produced by the weight load. This behaviour seemed consistent with in situ measurements of the wall's distortions, i.e. the wall suffered a strong buckling that was emphasized on its uppermost part.
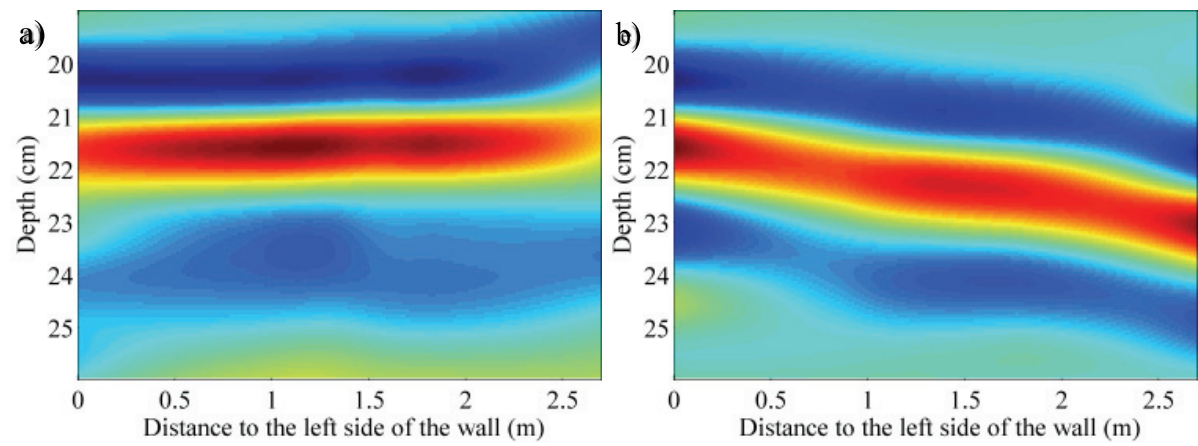

Fig. 5. Background at the opposite side of the wall for different values of the load. Wall 1 , row 6 ; a) no load; b) 80 metric tons (mt.) load.

\subsection{Analysis of the Wall with Imperfections (Backscattered Components)}

For the purpose of flaw detection, some algorithms were implemented in order to highlight the discontinuities (typically due to changes in the material) in the radargrams [10]. These methods, as seen on previous sections, were: background removal, depth resolution enhancing, Kirchhoff migration and improvement of the contrast in the B-scan. Fig. 6 shows the radargram obtained after this processing.

Fig. 6 corresponds to the radargram of row 2 with a load of $80 \mathrm{mt}$. A nook and a crack in the wall can be visually detected. Note the difference in perceived amplitude between both flaws. This can be explained by analysing the geometry of the flaws. The straight geometry of the crack accentuates the reflection of the waves producing higher amplitude values in the received signal. On the contrary, the nook is irregularly-shaped and rough-edged, which 
attenuates the reflections of the incident waves in the flaw scatters. Thus, received signal values for nook location are lower than those measured at the location of the crack.

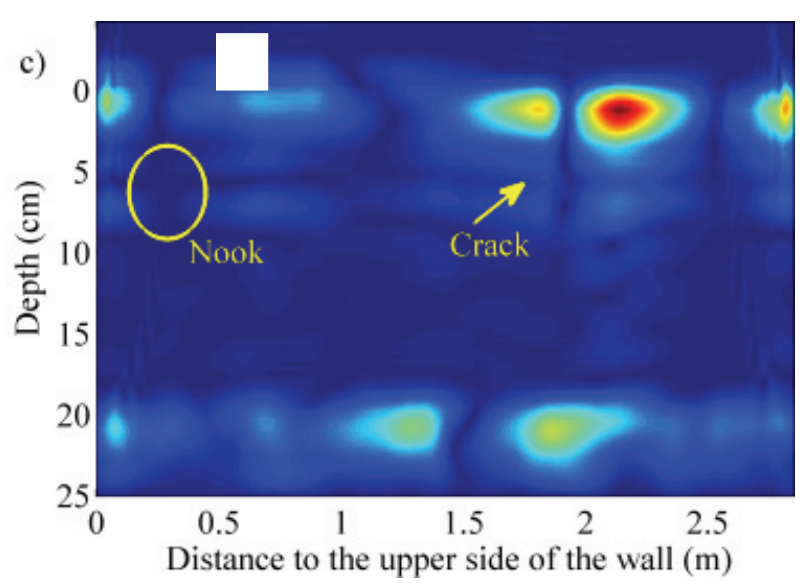

Fig. 6. Processed radargram with the imperfections highlighted.

The detection results were better for high load values. The reflection of incident waves from the flaws is strengthened with the increase in compression, which allows a better definition of the shapes of the imperfections in the radargrams.

\section{Conclusion}

The proposed method for the separation of background and backscattered components using ICA has demonstrated accurate separation in GPR signals from historic ashlar masonry walls. This has allowed enhanced images of the wall were obtained. The auscultation of historical masonry walls with ground-penetrating radar (GPR) has proved to be effective for the detection of imperfections and the characterization of walls under load. It was possible to detect flaws with millimetre sizes and variations in the interfaces between ashlars and mortar caused by the effect of the compression suffered under load.

Acknowledgments. This work has been supported by the Generalitat Valenciana under grant PROMETEO/2010/040, and the Spanish Administration and the FEDER Programme of the European Union under grant TEC 2008-02975/TEC.

\section{References}

1. Salazar, A., Unió, J.M., Serrano, A., Gosalbez, J.: Neural networks for defect detection in non-destructive evaluation by sonic signals. Lecture Notes in Computer Science, vol. 4507, pp. 638-645 (2007)

2. Salazar, A., Vergara, L., Llinares, R.: Learning material defect patterns by separating mixtures of independent component analyzers from NDT sonic signals. Mechanical Systems and Signal processing 24 (6), 1870-1886 (2010) 
3. Zhao, A., Jiang, Y., Wang, W.: Exploring Independent Component Analysis for GPR Signal Processing. In: Progress In Electromagnetics Research Symposium 2005, pp. 750-753. The Electromagnetics Academy, Cambridge, USA (2005)

4. Abujarad, F., Omar, A.: Comparison of Independent-Component Analysis (ICA) Algorithms for GPR Detection of Non-Metallic Land Mines. In: Bruzzone, L. (ed.), Image and Signal Processing for Remote Sensing XII. In: Proceedings of SPIE, vol. 6365, pp. 636516.1-636516.12, SPIE, Bellingham, USA (2006)

5. Liu, J.X., Zhang, B., Wu, R.B.: GPR Ground Bounce Removal Methods Based on Blind Source Separation. In: Progress In Electromagnetics Research Symposium 2006, pp.256-259. The Electromagnetics Academy, Cambridge, USA (2006)

6. Verma, P.K., Gaikwad, A.N., Sigh, D., Nigam, M.J.: Analysis of Clutter Reduction Techniques for Through Wall Imaging in UWB Range. In: Progress In Electromagnetics Research B 2009, vol. 17, pp. 29-48. The Electromagnetics Academy, Cambridge, USA (2009)

7. Salazar, A., Vergara, L., Serrano, A., Igual, J.: A General Procedure for Learning Mixtures of Independent Component Analyzers. Pattern Recognition 43 (1), 69-85 (2010)

8. Cardoso, J.F., Souloumiac, A.: Blind beamforming for non Gaussian signals. IEE Proceedings-F 140 (6), 362-370 (1993)

9. Ziehe, A., Muller, K.R.: TDSEP - An Efficient Algorithm for Blind Separation Using Time Structure. In: Proceedings of the Eighth International Conference on Artificial Neural Networks ICANN'98, Perspectives in Neural Computing, pp. 675-680 (1998)

10. Reynolds, J.M.: An Introduction to Applied and Environmental Geophysics. Wiley (1997)

11. Igual, J., Camacho, A., Vergara, L.: A blind source separation technique for extracting sinusoidal interferences in ultrasonic non-destructive testing. Journal of VLSI Signal Processing 38, 25-34 (2004)

12. Salazar, A., Gosalbez, Igual, J., Llinares, R., Vergara, L.: Two applications of independent component analysis for non-destructive evaluation by ultrasounds. Lecture Notes in Computer Science, vol. 3889, pp. 406-413 (2006)

13. Raghavan, R.S.: A Model for Spatially Correlated Radar Clutter. IEEE Trans. on Aerospace and Electronic Systems 27, 268-275 (1991)

14. Salazar, A., Vergara, L.: ICA mixtures applied to ultrasonic nondestructive classification of archaeological ceramics. EURASIP Journal on Advances in Signal Processing, vol. 2010, Article ID 125201, 11 pages, (2010). doi:10.1155/2010/125201

15. Salazar, A., Vergara, L., Miralles, R.: On including sequential dependence in ICA mixture models. Signal Processing 90 (7), 2314-2318 (2010) 